\title{
ESTRATEGIA VISUAL EN LA GENERALIZACIÓN DE PAUTAS LINEALES
}

\author{
GARCÍA CRUZ, JUAN ANTONIO y MARTINÓN, ANTONIO \\ Universidad de La Laguna.
}

\section{SUMMARY}

In this paper the role played by the drawing included in linear generalizing problems is outlined. The main focus is on the generalization developed by secondary education students. The research has been conducted over a population of 13 to 18 year old students. A visual strategy is identified and some modalities described. The Dörfler theoretical framework for the process of generalization is used to analize the students behaviour. Finally we conclude with some didactical remarks.

\section{INTRODUCCIÓN Y MARCO TEÓRICO}

Las pautas numéricas constituyen uno de los temas que se encuentran en los currículos. La percepción de esas pautas por los alumnos, su utilización para resolver problemas no triviales y su ulterior generalización se consideran tareas relevantes en la educación secundaria. Así, en el Curriculum and Evaluation Standars for School Mathematics norteamericano (NCTM, 1989, p. 98) encontramos «el currículo debe incluir la exploración de pautas y funciones para que los estudiantes sean capaces de describir, extender, analizar y crear una amplia gama de pautas» y también «usar pautas y funciones para representar y resolver problemas». Análogamente, en el Diseño Curricular Base español (DCB, 1989, p. 512) encontramos que el estudio de fenómenos y gráficos lineales y cuadráticos es tema de estudio para los alumnos de secundaria. Además, «identificar y describir regularidades, pautas y relaciones conocidas en conjuntos de números y formas geométricas similares» es uno de los criterios de evaluación para la enseñanza secundaria obligatoria (Real Decreto, 1991).

Las pautas y su relación con determinadas habilidades propias de la resolución de problemas, como es exami- nar casos especiales, organizar la información de forma sistemática, establecer conjeturas y generalizar, entre otras, ha sido objeto de algunas publicaciones (Mason et al., 1982; Swan, 1984). Además, la investigación de pautas permite a los estudiantes desarrollar la comprensión de conceptos y relaciones matemáticas importantes, también comprender las funciones, pues cuando se estudian las relaciones entre las cantidades en problemas con pautas surge el conocimiento entre importantes relaciones matemáticas y las funciones (Phillips 1991, p. 1). En todas estas publicaciones se pone énfasis en la relación que existe entre la percepción y extensión de pautas, ya sean numéricas o gráficas, su relación con el concepto de función y los procesos de abstracción y generalización, tan importantes en la resolución de problemas.

Algunos trabajos de investigación recientes se han dedicado a identificar y clasificar las estrategias utilizadas por los alumnos en los denominados problemas de generalización lineal, los cuales se caracterizan por los siguientes aspectos: se describe una situación que contiene en el enunciado un dibujo o diagrama que proporciona los primeros términos $f(1), f(2), f(3)$... de una sucesión 
aritmética, y se pide a los alumnos el cálculo de $f(n)$ para $n$ «pequeño» (generalización próxima) y «grande» (generalización lejana).

En Stacey (1989) se informa de los métodos, y de la consistencia en el uso del método, utilizados por alumnos de edades comprendidas entre 9 y 13 años en tareas de generalización lineal. Stacey informa que los alumnos más experimentados muestran, en su explicación escrita, cierta relación entre la pauta numérica y la pauta visual; es decir, se nota que el dibujo juega un cierto papel en el método empleado por los alumnos en la resolución de las tareas (p. 157), pero no profundiza en el estudio detallado del papel jugado por el dibujo.

En los trabajos de Orton y Orton (1994 y 1996) se utilizan mayoritariamente tareas con pautas lineales y cuadráticas, acompañados también de diagramas ilustrativos en el caso del primero de los trabajos aquí citados. En particular, en ese primer trabajo, se informa sobre un estudio llevado a cabo con alumnos de edades comprendidas entre los 9 y 13 años, en el que se utilizaron palillos. Se esperaba que el uso de tal material en la experiencia serviría de ayuda a los alumnos. Sin embargo, los autores informan que, una vez que los números asociados a los diagramas se hicieron explícitos, los alumnos abandonaron el trabajo con palillos y se concentraron exclusivamente en las pautas numéricas.

En Redden (1994) se utiliza la taxonomía SOLO (Structure of the Observed Learning Outcome, Biggs y Collis, 1982) en la clasificación de las respuestas de los alumnos ante tareas de pautas lineales, señalándose que ciertas respuestas de los alumnos están claramente influenciadas por el diagrama que acompaña al ítem (p. 92). Ya que el propósito del investigador era clasificar las respuestas en el modo simbólico concreto y no en el modo icónico, no consideró necesario prestar atención, en tal clasificación, a que las respuestas que desarrollaban los alumnos estuviesen influidas de alguna forma por el diagrama, aunque reconocía explícitamente que el diagrama podría estar influyendo en cierta manera (p. 90).

También Taplin (1995) utilizó la taxonomía SOLO como marco teórico para la clasificación de las respuestas de alumnos de edades comprendidas entre 12 y 13 años. Los ítems empleados se acompañaban de diagramas ilustrativos y además se utilizó material concreto para facilitar la tarea a los alumnos y estudiar el comportamiento de los mismos en varios formatos: modelización con material concreto, diagramas y texto. En particular, los diagramas se utilizaron para proveer de material adecuado a los estudiantes con tendencias visuales o pictóricas (p. 44). Sin embargo, no se señala explícitamente el uso que del diagrama hacen los alumnos en la construcción de la generalidad.

Castro (1995) plantea la viabilidad de las configuraciones puntuales como sistema de representación de los números naturales y como medio adecuado para visualizar y analizar secuencias. A través de una secuencia de tareas estructuradas y combinando otros dos sistemas de representación, se estudia la potencia de tales configuraciones para expresar relaciones y propiedades numéricas descubiertas por los alumnos (13 a 14 años). Concluye que tal sistema gráfico-intuitivo es ampliamente utilizado por los alumnos, aunque se detectan ciertos problemas en su uso. Por ejemplo, «al representar varios términos de una secuencia mediante configuración puntual, se pueden analizar sus términos mediante diferentes desarrollos aritméticos y, por tanto, obtener expresiones algebraicas distintas, aunque equivalentes del término general de la sucesión. La representación puntual del término general de una sucesión necesita de la ayuda del profesor; se trata de un conocimiento de los que Piaget denomina social, que necesita ser transmitido» (p. 308).

El objeto de estudio de las anteriores investigaciones, por lo general, ha sido clasificar las respuestas y establecer los niveles de generalización alcanzados por los alumnos. Es decir, creemos que se ha prestado poca atención al análisis del proceso mediante el cual los alumnos elaboran la generalización, habiéndose focalizado el esfuerzo en la clasificación del resultado de ese proceso.

Por nuestra parte (García Cruz y Martinón, 1996a, 1996b), hemos señalado parcialmente el papel jugado por el diagrama en la generalización de pautas lineales. Nuestra intención es profundizar en el análisis de ese papel y en este trabajo presentamos algunos resultados. Partimos de la hipótesis de que en el desarrollo y establecimiento de la generalización por parte de muchos alumnos juega un papel esencial el diagrama que acompaña a las situaciones. Desde este supuesto formulamos las siguientes cuestiones, objeto de nuestra investigación:

Cuestión 1: ¿Qué papel juega en el proceso de generalización el diagrama que acompaña a la tarea?

Cuestión 2: ¿Podemos esperar un comportamiento diferente en los alumnos según la edad y el nivel de instrucción recibido, entre aquéllos que usan el diagrama en el proceso de generalización?

En este trabajo estamos interesados en la forma en la que los alumnos establecen la generalización mediante un proceso en el cual el dibujo juega un papel esencial. En Presmeg (1986, p. 298) se define estrategia visual como el método de solución en el que son esenciales ciertas imágenes mentales, con o sin apoyo de un diagrama. Usamos ese término en sentido algo más restringido y denominamos estrategia visual al procedimiento de obtención de una regla general de cálculo para $f(n)$ en el que el dibujo juega un papel esencial.

El marco teórico sobre la generalización adoptado en nuestro trabajo es el de la generalización operativa desarrollado por Dörfler (1991), quien así ha modelado con detalle el proceso denominado por Piaget abstracción reflexiva, por medio del cuál se desarrolla y construye la generalización. En este modelo se presta especial atención al proceso de abstracción constructiva, al papel que juegan las acciones, los elementos de la acción y el establecimiento y simbolización de relaciones inva- 


\section{INVESTIGACIÓN DIDÁCTICA}

riantes en la construcción de la generalización. Según Dörfler, el proceso de generalización conlleva la construcción de variables, donde el término variable hace referencia tanto a un modelo cognitivo variable, en la cognición del sujeto, como a una parte del conocimiento objetivo, es decir, del contenido matemático. Dörfler añade que el estudio del comportamiento de los alumnos, el tipo de estrategia particular que desarrollan y las interpretaciones correspondientes deben confrontarse con la forma estándar de presentación y formulación del conocimiento matemático subyacente; de tal confrontación deben surgir elementos didácticos que sirvan de apoyo para la práctica docente.

\section{METODOLOGÍA}

\section{Cuestionario}

En el estudio hemos utilizado las dos siguientes tareas, que son las mismas que fueron usadas por Stacey (1989):

Tarea de la escalera
Ambas tareas resultan ser útiles como instrumentos para poder responder a la cuestión 1: ¿Qué papel juega en el proceso de generalización el diagrama que acompaña a la tarea? En efecto, se trata de tareas en las que es necesario que el alumno realice una generalización para responder a las cuestiones E5 y A2, aunque es posible que tal generalización se inicie en las respuestas a las cuestiones E4 y A1.

La tarea de la escalera comienza con tres cuestiones introductorias (E1, E2 y E3), que sirven a los alumnos de «calentamiento», y a continuación se les pide una generalización próxima (palillos necesarios para una escalera de tamaño 20, cuestión E4) y una generalización lejana (palillos necesarios para una escalera de tamaño 1.000, cuestión E5).

La tarea del árbol de Navidad no tiene cuestiones introductorias, sólo las cuestiones de generalización próxima (A1) y lejana (A2).

En este contexto, el término generalización próxima hace referencia a una cuestión que puede ser resuelta mediante un recuento directo sobre un dibujo construido al efecto o mediante la extensión de la sucesión numérica correspondiente; el término generalización lejana hace referencia a una cuestión que no se puede abordar o es

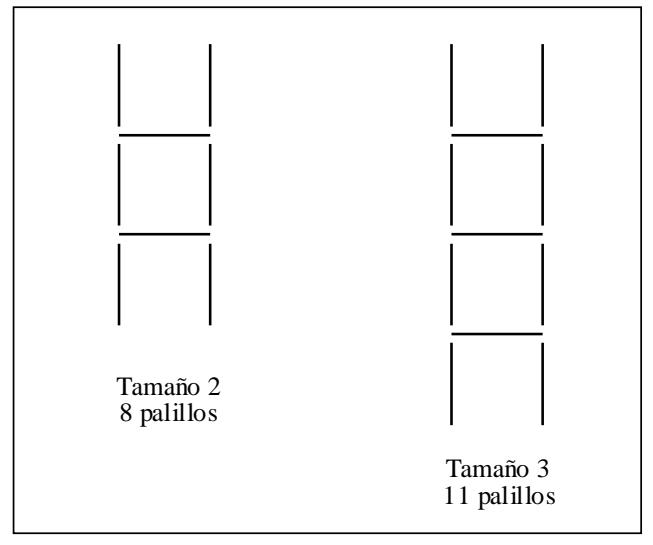

Tarea del árbol de Navidad

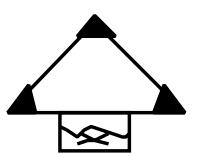

Tamaño 1

3 luces

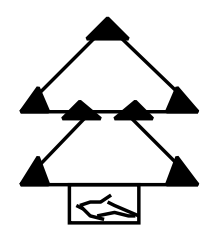

Tamaño 2

7 luces

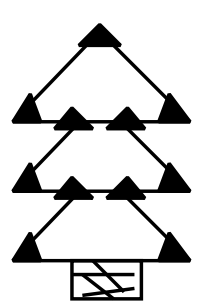

Tamaño 3

11 luces
E1: ¿Cuántos palillos serán necesarios para una escalera del mismo tipo con 4 peldaños?

E2: ¿Cuántos palillos serán necesarios para una escalera del mismo tipo con 5 peldaños?

E3: Sabemos que se necesitan 335 palillos para una escalera con 111 peldaños. ¿Cuántos palillos serán necesarios para una escalera del mismo tipo con 112 peldaños?

E4: ¿Cuántos palillos serán necesarios para una escalera del mismo tipo con 20 peldaños?

E5: ¿Cuántos palillos serán necesarios para una escalera del mismo tipo con 1.000 peldaños?

Explica tus respuestas.
A1: ¿Cuántas luces serán necesarias para un árbol de Navidad de tamaño 20 ?

A2: ¿Cuántas luces serán necesarias para un árbol de Navidad de tamaño 100 ?

Explica tus respuestas. 
difícil hacerlo por los procedimientos paso a paso descritos para la generalización próxima (Stacey, 1989).

La prueba se pasó en un tiempo máximo de una hora y se les pidió, tanto por escrito como oralmente, al comienzo y durante la prueba, que explicaran detenidamente cómo habían resuelto las diferentes cuestiones. En primer lugar, los alumnos realizaron la tarea de la escalera y una vez recogida se les pasó la tarea del árbol de Navidad. Esto se hizo así para estudiar si los alumnos eran consistentes en la estrategia utilizada al pasar de una tarea a otra.

El propósito del diagrama que acompaña a las dos tareas es que la pauta visual-geométrica ayude a los alumnos a percibir la pauta numérica. Teniendo en cuenta las dos cuestiones que nos hemos planteado en este trabajo, sólo analizamos en detalle aquellas respuestas dadas por los alumnos a las cuestiones de generalización próxima y lejana en las que usan una estrategia visual.

Resulta claro que las dos tareas responden a una pauta numérica lineal, tal como se denomina en la literatura didáctica. Se trata de sucesiones aritméticas de números naturales, $f(n)=a n+b(n=1,2,3 \ldots)$ con $a \neq 0$ y $b \neq 0$. Estas condiciones implican que la diferencia $a$ de la sucesión aritmética es un número natural y que $b$ es un número entero. Concretamente, en el caso de nuestras dos tareas, tenemos $f(n)=3 n+2$ en la escalera y $f(n)=$ $4 n$ - 1 en el árbol de Navidad. La presentación de la situación consiste en dar algunos de los primeros términos de la sucesión (dos en la escalera y tres en el árbol de Navidad). Debemos hacer notar que la condición $b \neq 0$ excluye las sucesiones del tipo $f(n)=a n$, que suelen denominarse de proporcionalidad directa, en las que el primer término $f(1)$ y la diferencia $a$ coinciden. Por otro lado, los alumnos pueden abordar este tipo de tareas desde el conocimiento de la expresión del término general $f(n)=f(1)+a(n-1)$ de una sucesión aritmética de diferencia $a$.

\section{Población}

El estudio se realizó sobre una población de 373 alumnos, cuyas edades y nivel educativo se recoge en la tabla I.

Tabla I

Número de alumnos según nivel educativo.

\begin{tabular}{llc} 
Edad (años) & Nivel & Alumnos \\
\hline $13-14$ & $8^{\circ}$ EGB & 62 \\
\hline $14-15$ & $1^{\circ}$ BUP & 59 \\
\hline $15-16$ & $4^{\circ}$ ESO & 80 \\
\hline $15-16$ & $2^{\circ}$ BUP & 52 \\
\hline $16-17$ & $3^{\circ}$ BUP & 62 \\
\hline $17-18$ & COU & 58 \\
\hline Total & & 373
\end{tabular}

Todos los alumnos habían seguido una instrucción tradicional y, por lo tanto, no estaban familiarizados con este tipo de tareas, salvo los alumnos del nivel $4^{\circ}$ de ESO. Estos alumnos se ubican en la nueva ordenación educativa en España y dentro de su currículo se da mucha importancia al trabajo con tareas no rutinarias, al empleo del razonamiento y a la explicación de las estrategias de solución empleadas. A los alumnos de $2^{\circ}$ de BUP se les pasó la prueba dos meses después de haber recibido enseñanza explícita sobre sucesiones aritméticas, y los alumnos de $3^{\circ}$ de BUP y COU habían recibido esta instrucción un año y dos años antes, respectivamente. En cuanto a los centros, dos eran urbanos, dos semiurbanos y uno rural. Los grupos de alumnos fueron seleccionados aleatoriamente en sus respectivos centros, de entre los grupos del mismo nivel que, a juicio de sus profesores, resultaban heterogéneos respecto de las habilidades matemáticas.

En ciertos casos, los dibujos fueron alterados de la siguiente manera: en el dibujo de la escalera se unieron los palillos y en el del árbol de Navidad se alinearon las luces. Tratamos con ello de analizar si una distinta configuración influiría en las respuestas. Se pasaron cuatro pruebas diferentes, cada una de las cuales fue respondida por un número similar de alumnos en cada uno de los niveles, tal como se indica en la tabla II.

Tabla II

Número de alumnos según modalidad de las pruebas.

\begin{tabular}{lccc} 
& Luces alineadas & Luces no alineadas & Total \\
\hline Palillos no unidos & 96 & 94 & 190 \\
\hline Palillos unidos & 92 & 91 & 183 \\
\hline Total & 188 & 185 & 373 \\
\hline
\end{tabular}

\section{RESULTADOS}

\section{Respuestas de los alumnos}

Las respuestas de los alumnos se han clasificado de la siguiente manera: en blanco (no hay respuesta), recuento (se cuenta el número de palillos o de luces sobre un dibujo que se corresponde con el tamaño del objeto en cuestión, o bien se prolonga numéricamente la sucesión hasta el término pedido), proporcional (se realiza un razonamiento de tipo proporcional, por ejemplo, usando la «regla de tres») y lineal (la respuesta se adapta, de alguna forma, a un esquema del tipo $a n+b$ ). En la tabla III se resume el número de respuestas de cada clase ante las cuestiones de generalización próxima y lejana, y también se incluye el número de alumnos que dan una respuesta lineal usando una estrategia visual. 
Tabla III

Número de alumnos según el tipo de respuesta.

\begin{tabular}{rrrrrrc} 
& B & R & W & L & EV & \% EV/L \\
\hline E4 & 47 & 44 & 136 & 146 & 63 & 43 \\
\hline E5 & 69 & 0 & 155 & 149 & 63 & 42 \\
\hline A1 & 56 & 14 & 86 & 217 & 119 & 55 \\
\hline A2 & 56 & 2 & 103 & 212 & 115 & 54 \\
\hline
\end{tabular}

(B: en blanco; R: recuento; W: proporcional; L: lineal; EV: estrategia visual en lineal)

Aunque no es objeto de este trabajo, puede apreciarse que las cuestiones sobre la escalera reciben un número de respuestas proporcionales similar a las lineales, mientras que en el árbol de Navidad hay un número muy superior de respuestas lineales.

Las respuestas de tipo lineal las hemos clasificado en las tres siguientes categorías:

L1: La respuesta corresponde a la estructura simbólica $f(n)=a n+b$, con $b \neq 0$ y $a$ la diferencia. Se trata de una respuesta de tipo funcional.

L2: Para el cálculo se hace uso explícito de expresiones del tipo $f(n)=d(n-m)+f(m), m<n, m \geq 1$. Se trata de una respuesta de tipo recursivo-funcional.

L3: Las expresiones para el cálculo son diferentes de las anteriores y en ellas no aparece explícitamente la diferencia constante.

Como hemos indicado, aquí sólo estamos interesados en las respuestas de tipo lineal que usan una estrategia visual. Con el fin de distinguir las diferentes modalidades de estrategia visual que los alumnos usan en cada una de las categorías de respuestas de tipo lineal, hemos diferenciado con un segundo dígito (L11, L12...) esas diferentes modalidades.

¿Cuándo consideramos que un alumno ha usado una estrategia visual? Como ya indicamos, siguiendo a Presmeg (1986), entendemos que un alumno ha usado una estrategia visual si, en el procedimiento de obtención de la regla de cálculo, el dibujo juega un papel esencial. Con el fin de precisar esto, distinguimos entre las diversas categorías de respuestas lineales.

En las categorías L1 y L2, consideramos que una respuesta es el resultado de una estrategia visual siempre que en la explicación exista una referencia clara a elementos del dibujo que no se encuentren en los enunciados de la tareas. Según este criterio, las explicaciones que incluyen sólo las palabras tamaño, árbol o escalera, que son palabras que aparecen en los enunciados, no las consideramos incluidas en la estrategia visual. Por otro lado, cuando en la explicación se utilizan palabras como partes laterales, líneas laterales, partes de abajo, partes de arriba, cúspide, triángulo, nivel, capa, punta de arriba, pisos uno debajo del otro, medios triángulos y similares, que no aparecen explícitamente en los enunciados y que indican claramente una referencia a elementos de la escalera o del árbol, hemos considerado que tal respuesta sí se corresponde con una estrategia visual.

En la categoría L3 fijamos un criterio no tan restrictivo como en L1 y L2, ya que hemos considerado que en una respuesta juega un papel esencial el dibujo cuando la explicación más plausible, para el cálculo efectuado, conlleva referencias a los elementos del mismo y no hay otra posible explicación alternativa que haya sido constatada en respuestas puramente numéricas dadas por otros alumnos. Un ejemplo lo constituye la modalidad L31, que se expone a continuación, y cuya expresión para el cálculo es fácilmente deducible a partir del dibujo y no a partir de la sucesión numérica.

\section{Tarea de la escalera}

En esta tarea, 68 alumnos (18\% del total de 373 ) hacen referencia expresa al diagrama que acompaña a la situación al calcular el total de palillos necesarios en alguna de las cuestiones E4 y E5 planteadas. En sus explicaciones, dadas por escrito, utilizan por lo general el dibujo de mayor tamaño $(n=3)$, donde introducen signos pictóricos para agrupar elementos, y mediante sentencias verbales expresan la relación entre los elementos del mismo. Una vez analizadas las respuestas, y no habiendo encontrado diferencias significativas respecto de las dos modalidades del dibujo (palillos separados o juntos), las hemos clasificado bajo las dos siguientes modalidades:

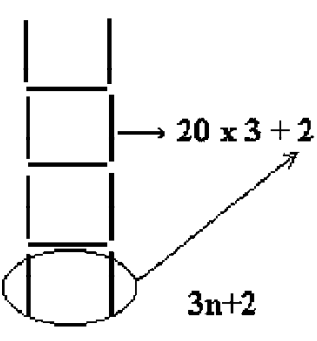

Modalidad L11

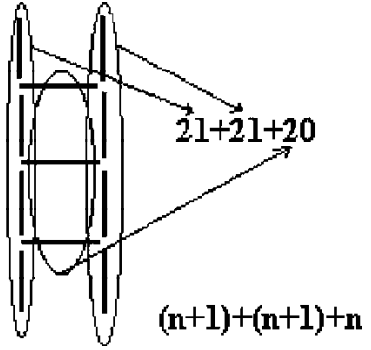

Modalidad L31
Modalidad L11: Consiste en separar dos palillos en la base, o en lo alto, y considerar el resto de la escalera formada por una sucesión de elementos compuestos a su vez por tres palillos, uno horizontal y dos verticales. Si la escalera tiene tamaño 20 (peldaños), el número de palillos será, por consiguiente, igual a $3(20+2$. Las respuestas de esta modalidad se corresponden con un esquema del siguiente tipo: si la escalera tiene tamaño $n$ (peldaños), entonces estará formada por $3 n+2$ palillos. Por tanto, la estructura aritmético-algebraica de esta modalidad corresponde a la forma canónica de la pauta lineal, es decir, $f(n)=a n+b$, que resulta claramente funcional. Sin embargo, al obtener el alumno tal expre- 
sión a partir del dibujo, hemos de pensar que tiene un marcado carácter recursivo: el término inicial está constituido por los dos palillos de la base, o de la parte superior de la escalera, y cada término se construye añadiendo tres palillos, uno de los cuales corresponde con un peldaño. Por otro lado, tal proceso recursivo no tiene un equivalente en la sucesión, donde claramente el primer término corresponde a la escalera formada por cinco peldaños. Luego, el dibujo puede inducir en los alumnos un tipo de respuesta que en esencia es de cualidad recursiva aunque podría confundirse con una de tipo funcional.

Modalidad L31: Consiste en separar la escalera en tres bloques diferenciados, de los que dos corresponden a las columnas laterales de palillos verticales y la otra, a los palillos horizontales o peldaños. Si la escalera tiene tamaño 20 (peldaños) tendremos $20+1$ por cada lateral, más los 20 centrales; es decir, $(20+1)+(20+1)+20=$ 62 palillos. El esquema subyacente en estas respuestas es el siguiente: si la escalera tiene tamaño $n$ (peldaños), entonces estará formada por $(n+1)+(n+1)+n$ palillos. La respuesta es claramente funcional, pero no se utiliza la diferencia constante como elemento constitutivo de la misma. Realmente la respuesta se construye a partir de dos relaciones funcionales: los palillos-peldaños $(n)$ y los palillos-laterales $(n+1)$ contados dos veces.

El total de alumnos que usan la estrategia visual en alguna de las cuestiones E4 o E5, por nivel educativo y modalidad, se muestran en la tabla IV, donde el porcentaje hace referencia al total de alumnos de cada nivel educativo que constituyen la población estudiada.

Tabla IV

Alumnos por modalidad en la tarea de la escalera.

\begin{tabular}{lcccc} 
& \multicolumn{2}{c}{ Modalidades } & \multicolumn{2}{c}{ Totales } \\
\hline Niveles & L11 & L31 & Alumnos & $\%$ \\
\hline $8^{\circ}$ EGB & 1 & 2 & 3 & 5 \\
\hline $4^{\circ}$ ESO & 20 & 6 & 26 & 33 \\
\hline $1^{\circ}$ BUP & 9 & 7 & 16 & 27 \\
\hline $2^{\circ}$ BUP & 2 & 1 & 3 & 6 \\
\hline $3^{\circ}$ BUP & 2 & 4 & 6 & 10 \\
\hline COU & 10 & 4 & 14 & 24 \\
\hline Totales & 44 & 24 & 68 & 18 \\
\hline
\end{tabular}

Debemos señalar que no hay alumnos que usen dos modalidades distintas en las cuestiones de esta tarea de la escalera.

\section{Tarea del árbol de Navidad}

En esta tarea, 119 alumnos (32\%) hacen referencia expresa al diagrama que acompaña a la situación al calcular el total de luces necesarias en alguna de las dos cuestiones planteadas. Una vez analizadas las respuestas de los alumnos, hemos clasificado y agrupado las mismas bajo las modalidades que ahora se indican. Las dos primeras, L21 y L22, fueron usadas en las tareas con luces alineadas, salvo 2 alumnos que usaron la L22 con las luces no alineadas, mientras que las restantes fueron utilizadas cuando las luces no estaban alineadas.

Modalidades L21 y L22: En ambas el esquema es muy similar y consiste en descomponer el árbol en copa, centro y base. En la modalidad L21, la copa consta de 5 luces; el centro está formado por un número variable de capas, según el tamaño del árbol, cada una con 4 luces; y la base está formada por 2 luces. La modalidad L22 difiere de la L21 en que la copa está formada por 1 luz solamente. Si ahora el árbol tiene tamaño 20, en la modalidad L21 el número total de luces necesarias será $5+4(18+2$, mientras que en la modalidad L22 el número total de luces será $1+4(19+2$. Las respuestas corresponden a los esquemas siguientes: si el árbol tiene tamaño $n$, entonces se necesitan $5+4(n-2)+2$ y $1+$ $4(n-1)+2$ luces, para las modalidades L21 y L22, respectivamente.

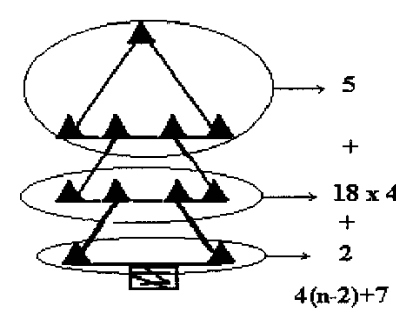

Modalidad L21

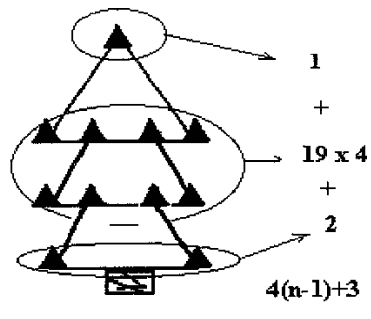

Modalidad L22
Modalidad L23: Consiste en considerar el árbol formado por una copa con 3 luces y por elementos variables, según el tamaño del árbol, compuestos cada uno por 4 luces. Si el árbol tiene tamaño 20 , tendrá un número de luces igual a 4(19+3; responde al siguiente esquema: si el tamaño es $n$, entonces se tendrá un número de luces igual a $4(n-1)+3$.

Modalidad L32: Consiste en agrupar las luces de tres en tres, dejando una columna en el centro del árbol formada por luces aisladas. Si el tamaño del árbol es 20, se tendrá un número de luces igual a $3(20+19$; el esquema es: si el árbol tiene tamaño $n$, entonces el número de luces será igual a $3 n+(n-1)$.

Modalidad L33: Esta modalidad es similar a la L11 de la escalera y consiste en agrupar las luces en columnas, cuya cantidad varía con el tamaño del árbol, y en dejar una luz aislada en la copa del árbol. Si el árbol tiene tamaño 20, entonces el número de luces necesario será $20+20+19+19+1$; el esquema es el siguiente: si el árbol tiene tamaño $n$, entonces el número de luces será $n+n+(n-1)+(n-1)+1$ 
Modalidad L12: Difiere de la modalidad L23 en que se añade una luz a la copa para que ésta contenga un número de luces igual a las que tiene cada elemento de la parte variable; a continuación se multiplica el tamaño por 4 y se resta 1 (la luz que se había añadido) al resultado final. Se sigue que, si el tamaño del árbol es 20, entonces, el número de luces es 4(20 - 1; por lo tanto, el esquema es: si el tamaño del árbol es $n$, entonces el número de luces es $4 n-1$.

En esta tarea, las modalidades L21, L22 y L23, que son evidentemente recursivas en su formulación aritmética y algebraica, coinciden con un procedimiento recursivo de construcción del dibujo. En las dos primeras, el elemento de partida es el número de objetos (luces) que hay en la copa y base del árbol; en la última, este elemento lo forman las tres luces de la copa. Esta última construcción puede ser visualizada construyendo el árbol desde el primer elemento hacia abajo, añadiendo partes idénticas que contienen cuatro luces. Todas estas formulaciones recursivas a partir del dibujo tienen su correspondiente equivalencia en la sucesión numérica, hecho que no ocurre con la modalidad L11 de la tarea de la escalera, como ya señalamos anteriormente. Es de notar que las diferentes modalidades se usan, o bien con luces alineadas, o bien con no alineadas, de forma que no hay modalidades de la estrategia visual comunes a las dos formas de presentar las luces. Hay, pues, una clara influencia de que las luces estén o no alineadas en la modalidad que se usa.

En la tabla V se indica el número de alumnos que usan la estrategia visual en alguna de las cuestiones A1 o A2, por nivel educativo y modalidad.
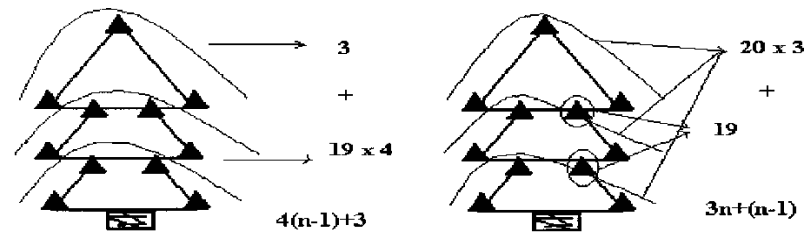

Modalidad L23

Modalidad L32

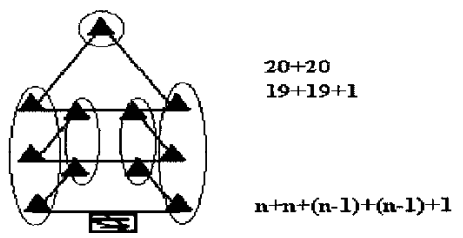

Modalidad L33
Tabla V

Alumnos según modalidad en la tarea del árbol de Navidad.

\begin{tabular}{lrrrrrrrrr}
\multicolumn{1}{c}{ Modalidades } \\
\hline Niveles & L21 & L22 & L23 & L12 & L32 & L33 & Alumnos & $\%$ \\
\hline $8^{\circ}$ EGB & 2 & 6 & 1 & 2 & 0 & 0 & 11 & 18 \\
\hline $4^{\circ}$ ESO & 4 & 10 & 8 & 6 & 1 & 2 & 31 & 39 \\
\hline $1^{\circ}$ BUP & 1 & 10 & 3 & 4 & 0 & 0 & 18 & 30 \\
\hline $2^{\circ}$ BUP & 2 & 4 & 0 & 1 & 0 & 0 & 7 & 13 \\
\hline $3^{\circ}$ BUP & 4 & 6 & 9 & 1 & 1 & 1 & 22 & 35 \\
\hline COU & 7 & 6 & 11 & 4 & 1 & 1 & 30 & 52 \\
\hline Totales & 20 & 42 & 32 & 18 & 3 & 4 & 119 & 32 \\
\hline
\end{tabular}

Tal como ocurre en la tarea de la escalera, no hay alumnos que usen dos modalidades distintas en las cuestiones de esta tarea del árbol de Navidad.

\section{Análisis de los errores}

En la tarea de la escalera se observaron un total de 6 errores, correspondientes a 7 alumnos, y no se observaron diferencias apreciables entre las pruebas en las que los palillos se unieron respecto de las que éstos se mantuvieron como en el formato original. Concretamente:

Modalidad L31 (3 alumnos):

$$
\begin{aligned}
& 22+22(1 \text { alumno }) \\
& 22+22+20(1 \text { alumno }) \\
& 20+19+19(1 \text { alumno })
\end{aligned}
$$

Modalidad L11 (4 alumnos):

$$
\begin{aligned}
& 20 \times 2+2 \text { ( } 2 \text { alumnos }) \\
& 20 \times 3+5 \text { (1 alumno } \\
& 20 \times 2+4 \text { (1 alumno })
\end{aligned}
$$

En la tarea del árbol de Navidad hemos contabilizado 25 alumnos que cometieron errores. Aquí sí se aprecian diferencias entre las pruebas con las luces alineadas y las que no. Cuando las luces estaban alineadas, los errores cometidos fueron los siguientes (21 alumnos):

Modalidad L22 (19 alumnos):

$$
\begin{aligned}
& 18 \times 4+3 \text { (13 alumnos) } \\
& 20 \times 4+3 \text { (4 alumnos) } \\
& 19 \times 4+1 \text { ( } 1 \text { alumno }) \\
& 18 \times 4+5 \text { (1 alumno) }
\end{aligned}
$$

Modalidad L21 (2 alumnos):

$$
18 \times 3+7 \text { (2 alumnos) }
$$

En las pruebas en las que las luces no estaban alineadas los errores fueron los siguientes (4 alumnos): 
Modalidad L12 (3 alumnos):

$20 \times 4$ - 2 (1 alumno)

$20 \times 4-3$ (1 alumno)

Modalidad L32 (1 alumno)

$$
20 \times 3+20 \text { (1 alumno) }
$$

En la modalidad L22 (19 alumnos), todos los errores se deben a una apreciación errónea entre el tamaño del objeto y el número de partes en las que se descompone el elemento variable del mismo, más que a una incompetencia aritmética. En las modalidades L23 y L33 no se contabilizaron errores.

Si comparamos el porcentaje relativo de respuestas atendiendo al diferente formato del diagrama, observamos que un $40 \%$ (25 alumnos de un total de 62) cometieron errores en las pruebas con las luces alineadas, mientras que sólo un $7 \%$ (4 alumnos de un total de 57) cometieron errores en las pruebas con las luces no alineadas. Además, el porcentaje de alumnos que utilizan el diagrama en su explicación es muy similar en ambas pruebas: $33 \%$ con luces alineadas (62 alumnos de un total de 188) y $31 \%$ con luces no alineadas (57 alumnos de un total de 185). Luego, nuestra decisión de modificar el dibujo alineando las luces parece que indujo a los alumnos a cometer un número mayor de errores.

\section{Uso de la estrategia visual}

Hasta ahora los datos presentados en este apartado se refieren al número de veces que se ha aplicado cada modalidad de la estrategia visual, pero nos parece interesante comprobar cómo se comportan los alumnos que emplean una u otra modalidad al pasar de una cuestión a otra dentro de una misma tarea, incluso entre las dos tareas.

La tabla VI muestra el número de alumnos que contestó a cada cuestión con referencia explícita al dibujo. Bajo la columna «Prueba» se recoge el total de alumnos que utilizaron la estrategia visual en las cuatro cuestiones. Bajo la columna «Totales» se recoge el total de alumnos que utilizaron esta estrategia para resolver, al menos, una cuestión.

Tabla VI

Alumnos que usan la estrategia visual.

\begin{tabular}{lrrrrrrrrr} 
& \multicolumn{2}{c}{ Escalera } & \multicolumn{2}{c}{ Árbol } & \multicolumn{2}{c}{ Prueba } & \multicolumn{2}{c}{ Totales } \\
\hline Niveles & E4 & E5 & A1 & A2 & Alumnos & $\%$ & Alumnos & $\%$ \\
\hline $8^{\circ}$ EGB & 3 & 1 & 11 & 11 & 0 & 0 & 14 & 23 \\
\hline $1^{\circ}$ BUP & 14 & 16 & 18 & 18 & 9 & 15 & 25 & 42 \\
\hline $4^{\circ}$ ESO & 26 & 24 & 31 & 28 & 13 & 16 & 44 & 55 \\
\hline $2^{\circ}$ BUP & 3 & 3 & 7 & 6 & 2 & 4 & 8 & 15 \\
\hline $3^{\circ}$ BUP & 5 & 6 & 22 & 22 & 6 & 10 & 22 & 35 \\
\hline COU & 12 & 13 & 30 & 30 & 10 & 17 & 34 & 59 \\
\hline Totales & 63 & 63 & 119 & 115 & 40 & 11 & 147 & 39 \\
\hline
\end{tabular}

En primer lugar, sospechamos que muchos más alumnos de los recogidos en la tabla VI emplearon la estrategia basada en el dibujo, aunque optaron por no dar ninguna explicación y se limitaron a consignar el resultado en cada cuestión.

El bajo porcentaje de alumnos de $2^{\circ}$ de BUP que utilizan la estrategia visual no debe sorprendernos, pues eran alumnos que habían sido instruidos dos meses antes sobre sucesiones aritméticas $\mathrm{y}$, al reconocer la pauta numérica como correspondiente a este tipo de situaciones, utilizaron mayoritariamente la expresión $f(n)=f(1)$ $+d(n-1)$. Sorprendentemente el mayor porcentaje de alumnos que utilizan el diagrama en la explicación de sus cálculos se obtuvo en los alumnos con mayor edad y nivel académico (COU). Algunos de estos alumnos, así como en $3^{\circ}$ de BUP, reconocieron la pauta como «aritmética» pero anotaron frases como «no recuerdo la fórmula a aplicar» y optaron por desarrollar una estrategia personal de recuento.

Si comparamos los alumnos de $1^{\circ}$ de BUP y $4^{\circ}$ de ESO, observamos que el porcentaje de alumnos que utilizaron esta estrategia en las cuatro cuestiones es similar (columna «Prueba» de la Tabla VI), siendo mayor en $4^{\circ}$ de ESO si se observa cuestión por cuestión y en el total de alumnos que a lo largo de la prueba utilizaron por lo menos una vez esta estrategia (última columna de la tabla VI). Esta diferencia podría ser debida, en parte, a la distinta metodología aplicada por el profesor en los diferentes niveles educativos, observación que señalamos en el apartado relativo a la población estudiada

La tabla VI muestra claramente la inconsistencia en el uso y empleo de esta estrategia visual a lo largo de toda la prueba, no así en cada tarea, en la cual los alumnos que utilizan esta estrategia visual la emplean, por lo general, en ambas cuestiones. Otro hecho puesto de relieve por la última columna de la tabla VI es el alto porcentaje de alumnos que utilizan, al menos una vez, tal estrategia al responder a alguna cuestión dentro de las dos tareas (39\% de la población total).

\section{DISCUSIÓN}

En este apartado profundizamos en lo que los alumnos hacen a la luz del marco de la generalización operativa (Dörfler, 1991). Concluimos con algunas sugerencias didácticas para la práctica en el aula.

\section{Acciones: visiones estática y dinámica}

La cuestión a resolver, cálculo de $f(n)$ para un cierto $n$, dirige la atención de los alumnos hacia los elementos del dibujo y el tamaño $n$ del objeto. A partir de ahí, los alumnos desarrollan una acción mental, que puede suponer o no una actividad física sobre el dibujo, cuyo esquema precisamos a continuación.

Las respuestas que efectivamente dieron los alumnos permiten inferir que hay dos clases de acciones diferen- 
tes, según contemplen el dibujo de forma estática o dinámica. Obtenemos así una primera clasificación de las acciones.

Cuando un alumno considera de forma estática el dibujo, imagina el objeto completo del tamaño requerido. Veamos un ejemplo. En la modalidad L22, ante la cuestión A1, cálculo de $f(20)$, el alumno imagina un árbol de tamaño 20 mirando el árbol de tamaño 3 que figura en el enunciado de la tarea. Entonces, la acción consiste en dividir el dibujo del árbol produciendo una partición en el conjunto de las luces, de forma que surgen tres grupos de luces: un grupo de 1 luz en la copa, un grupo en el centro formado por 19 subgrupos de 4 luces cada uno y un grupo de 2 luces en la base. La acción realizada sobre el árbol de tamaño 20 puede modificarse ligeramente para adecuarla a un árbol de cualquier tamaño, y para ello es suficiente variar el número de subgrupos de 4 luces.

Respuesta: $19(4=76+3=79$. El árbol de tamaño 20 está formado por 20 triángulos y en 19 de sus triángulos posee 4 luces, por lo que tiene 76, pero se le suman 3 más porque son las que van en las dos puntas del primer triángulo y en la punta del triángulo número 20.

Como se observa, la visión estática del dibujo lleva a la realización de una acción que produce un número constante de grupos, aunque los hay de dos clases: grupos de tamaño invariable al modificar el tamaño del árbol (uno en la copa y otro en la base) y un grupo cuyo tamaño varía en función del tamaño del árbol. Podemos simbolizar del siguiente modo el esquema de la acción correspondiente a la modalidad L22:

$$
\langle\text { invariable }\rangle+\langle\text { variable }\rangle+\langle\text { invariable }\rangle \text {. }
$$

Si consideramos la modalidad L31 de la escalera, habrá tres grupos variables:

$$
\langle\text { variable }\rangle+\langle\text { variable }\rangle+\langle\text { variable }\rangle \text {. }
$$

Lo que es común a los esquemas correspondientes a una visión estática es que el número de grupos es constante, en el sentido de que no varía con el tamaño del objeto.

Si el alumno contempla el dibujo de forma dinámica, entonces lo imagina creciendo desde uno de los tamaños que aparecen en el enunciado de la tarea hasta el tamaño superior por el que se le pregunta. Consideremos, de nuevo, el ejemplo de la modalidad L22. Hay alumnos que han imaginado el árbol de tamaño 3 como un árbol de tamaño 1 al que se le han añadido dos capas de 4 luces en el centro y, por tanto, mediante la acción de incorporar «niveles» de 4 luces, y dejando inalteradas la copa y la base, alcanzan el tamaño 20 o un tamaño superior.

Respuesta: $20 ; 3+(4 \cdot 19)=79$ luces. Si en el primer nivel hay tres, que al colocarle uno encima desaparece el de la punta, pero que aparece en el último nivel y sabemos que en el resto de los niveles hay cuatro.

Tendremos: 1 er nivel $-2+1$ (aparece en la punta del último nivel) resto — 4 . (núm. de niveles restantes).
En este caso, el número de grupos no es constante, depende del tamaño del árbol (en el árbol de tamaño 3 hay 4 grupos, en el de tamaño 20 hay 21 grupos), pero el número de luces en cada grupo no varía con el tamaño del árbol (1 luz en el grupo de la copa, 4 luces en los grupos del centro y 2 luces en el grupo de la base). El esquema de esta acción podemos simbolizarlo mediante:

$\langle$ invariable $\rangle+[\langle$ invariable $\rangle+\langle$ invariable $\rangle+\ldots]+$ $<$ invariable>.

En la modalidad L11 de la escalera el esquema de la acción correspondiente a una visión dinámica sería:

$\langle$ invariable $\rangle+[\langle$ invariable $\rangle+\langle$ invariable $\rangle+\ldots]$

En todos los esquemas de las acciones de una visión dinámica, el número de grupos depende del tamaño del objeto.

Resulta, por tanto, que la manera en que se produzca la visión del dibujo determina un tipo u otro de esquema de acción. La visión estática conduce a un número constante de grupos alguno de los cuales es variable, mientras que la visión dinámica lleva a un número no constante de grupos, teniendo todos ellos un número invariable de elementos. En ambos casos hay elementos (grupos o tamaño de grupos) variables e invariables.

Debe observarse que dentro de una misma modalidad de estrategia visual pueden darse ambos tipos de visiones del dibujo y, por tanto, las dos clases de acciones que hemos descrito. Ocurre así con la modalidad L22, usada antes como ejemplo en ambos casos.

\section{Acciones: esquemas con diferencia y sin diferencia}

Una segunda clasificación de las acciones y de los esquemas de las acciones tiene que ver con el papel que juega la diferencia $a$. En ciertos casos, los elementos variables se relacionan con la diferencia, mientras que en otros no es así. Es decir, tenemos dos tipos de esquema de la acción.

Esquema A: con diferencia. El elemento variable del esquema de la acción está directamente relacionado con la diferencia $a$ de la sucesión. Así ocurre en las modalidades L11 de la escalera y L12, L21, L22 y L23 del árbol de Navidad. En la visión estática, el grupo variable está formado por subgrupos de tamaño $a$ y, en la visión dinámica, los grupos que se van añadiendo tienen tamaño $a$. Consideremos, por ejemplo, la modalidad L11. Con una visión estática, el alumno produce una partición del tipo:

$$
<2>+<20(3>
$$

En una visión dinámica, los grupos que se añaden son de 3 elementos:

$$
\langle 2\rangle+\langle 3\rangle+\langle 3\rangle+\ldots
$$


En ambos supuestos la diferencia 3 juega un papel esencial.

Esquema B: sin diferencia. El elemento variable del esquema de la acción no está directamente relacionado con la diferencia $a$ de la sucesión, como ocurre en las modalidades L31 de la escalera y L32 y L33 del árbol de Navidad. Veamos el ejemplo de la modalidad L31. Con visión estática el alumno actúa de modo que se produce una partición como:

$$
\langle 21\rangle+\langle 20\rangle+\langle 21\rangle
$$

Desde una visión dinámica se tendrá:

$[\langle 2\rangle+\langle 1\rangle+\langle 1\rangle+\ldots]+[\langle 2\rangle+\langle 1\rangle+\langle 1\rangle+\ldots]+[\langle 2\rangle$ $+\langle 1\rangle+\langle 1\rangle+\ldots]$

En ninguno de los dos casos aparece la diferencia 3 de modo explícito, ya que lo que ocurre es que la sucesión de diferencia 3 se descompone en suma de tres sucesiones de diferencia 1. En la tabla VII se da el número de alumnos que utilizaron los distintos esquemas en cada prueba, referidos exclusivamente a los 40 que usaron la estrategia visual en las cuatro cuestiones E4, E5, A1 y A2.

Tabla VII

Esquemas de acción

\begin{tabular}{ccc} 
& Escalera & Árbol \\
\hline A & 25 & 38 \\
\hline B & 15 & 2 \\
\hline
\end{tabular}

\section{Invariante y generalización}

La ejecución de las acciones que hemos descrito permite establecer una relación entre el tamaño $n$ del objeto y el número de elementos $f(n)$ que lo constituyen. Las acciones confieren carácter constructivo a la generalización que se alcanza, pues está en la génesis del proceso que concluye en la obtención de la regla general para el cálculo del número de objetos $f(n)$ (palillos o luces), para $\operatorname{los} n$ (tamaño) que se indican.

En el ámbito de la estrategia visual, debe observarse que el establecimiento de la relación entre $n$ y $f(n)$ no se logra, al menos únicamente, mediante un proceso de inducción. La inducción supone la observación de cierta regularidad en varios casos particulares (que se dan al alumno en el enunciado) y de ahí se obtiene la relación general (Polya, 1966). Así, en el ejemplo de la escalera, el proceso de inducción que daría lugar a la expresión general se basaría en la observación de que:

$$
\begin{aligned}
& f(1)=2+1 \times 3, \\
& f(2)=2+2 \times 3, \\
& f(3)=2+3 \times 3,
\end{aligned}
$$

y de ahí se inferiría que $f(20)=2+20$ x 3 y que $f(1000)$ $=2+1000 \times 3$. En este caso, la inducción se produce a partir de la observación de expresiones adecuadas para $f(n)$.

Sin embargo, en el proceso que estamos describiendo, relativo a la estrategia visual, lo que realmente ocurre es que el alumno actúa sobre el objeto de un cierto tamaño para establecer una relación entre $n$ y $f(n)$, construyendo de ese modo la generalización. Casi nunca necesita dibujos del objeto de diferentes tamaños. Como ejemplo, observemos la modalidad L31. Las acciones realizadas sobre el dibujo conducen a descomponer la escalera en tres grupos: uno central, formada por los palillos horizontales (peldaños), y dos laterales formadas por los palillos verticales. Es suficiente, a partir del objeto de tamaño 3, imaginar el objeto de tamaño requerido y actuar sobre él a fin de obtener la expresión para el cálculo, como, por ejemplo, 20 (peldaños) + dos veces 21 (palillos verticales) cuando $n=20$.

Dörfler (1991) considera que para la construcción de una generalización es necesario el establecimiento de un invariante. En general, la relación invariante se establece en la cuestión de generalización próxima, utilizando el dibujo del objeto de tamaño 3 , y se extiende a la cuestión de generalización lejana. Nosotros consideramos que sólo aquellos alumnos que utilizan al menos dos veces la misma estructura para la regla de cálculo han establecido un invariante y, por lo tanto, han realizado una generalización. Por ejemplo, entendemos que se establece el invariante en la cuestión de la escalera si se usa la misma regla de cálculo en las cuestiones E4 y E5. Así, varios alumnos no han establecido el invariante: algunos no extienden el cálculo realizado en la cuestión de generalización próxima a la cuestión de generalización lejana ( 5 alumnos en la escalera y 4 en el árbol), mientras que otros hacen un recuento sobre un dibujo de tamaño adecuado en la generalización próxima y establecen una relación aritmética en la lejana ( 5 en la escalera y ninguno en el árbol). Es decir, el éxito en un cálculo concreto no garantiza la generalización de la regla.

La generalización tiene dos cualidades. La primera es una cualidad intensional, que se manifiesta en la estructura sintáctica y semántica de la regla de cálculo, abstraída en una cuestión concreta y para un cálculo concreto. Tal estructura, como ya hemos señalado, contiene elementos variables e invariables, y sus significados están claramente asociados a partes del dibujo. La segunda cualidad es la extensional, que se refiere al rango de los elementos variables. Tales cualidades son imprescindibles para el logro de una generalización dentro del marco teórico aquí adoptado.

Ahora cabe hacernos la siguiente pregunta: ¿Qué han generalizado los alumnos? A la luz de la discusión anterior sólo podemos concluir que se ha generalizado un procedimiento de cálculo; es decir, una regla para el cálculo dentro de una misma tarea, lo que podemos denominar generalización local. Para aquellos alumnos que han utilizado la misma relación invariante en ambas cuestiones dentro de una misma tarea, parece claro que la expresión aritmética para un cálculo específico, generalización próxima, refleja la estructura general de dicho 
cálculo y produce en los alumnos el desarrollo de una generalización intensional, comprobable al aplicar la misma relación invariante a la cuestión de generalización lejana. Este recuento generalizado muestra el aspecto procedimental del comportamiento cognitivo, puesto que establece un orden de los términos $f(n)$ de la sucesión, de modo que el correspondiente número natural $n$ (tamaño del objeto) se contempla como una especificación ordinal de las cantidades de la sucesión y se obtiene la relación variable entre el número $n$ y la cantidad correspondiente $f(n)$, siendo este comportamiento cognitivo un ejemplo de lo que Dubinsky y Harel (1992, p. 91) denominan concepto procedimental de función. Sin embargo, para establecer una entidad conceptual como es el objeto matemático $f(n)=a n+b$, es necesario variar los elementos que lo componen, extender el rango de referencia de los elementos y de sus símbolos (Dörfler, 1991).

Por tanto, se obtiene una relación entre $n$ y $f(n)$ en la que figura el par de parámetros concretos $a$ y $b$. Dentro de cada tarea, el par de parámetros $a$ y $b$ ha permanecido constante. Lo que podemos concluir es que los alumnos han abstraído un método general de recuento para cada tarea. En principio, ese esquema general de recuento es sólo de aplicación a cuestiones dentro de la tarea en la que se ha establecido. Por ello creemos que, mientras no se perciba el mismo esquema a través de una secuencia de tareas, lo que supone la variación de los parámetros $a$ y $b$, no es posible contemplar la similitud entre las diferentes estructuras de cálculo y, por lo tanto, reconocer esa estructura como similar a $f(n)=a n+b$, o equivalentes.

Ya hemos señalado que las reglas de cálculo generales tienen su origen en determinadas acciones sobre el dibujo y, de alguna forma, las relaciones entre las diferentes partes del dibujo y sus elementos se traducen en relaciones aritméticas entre los números. Pues bien, podemos ahora caracterizar con más precisión la noción de estrategia visual, y de sus diferentes modalidades, afirmando que se compone de las acciones particulares realizadas sobre el dibujo, de las relaciones entre elementos del mismo y de las relaciones aritméticas que se formulan mediante la regla general de cálculo, es decir, en el invariante establecido en el proceso de generalización. Con esta noción más precisa podremos profundizar aún más sobre el comportamiento de los alumnos en la secuencia de las dos tareas.

Estudiemos la transición entre ambas tareas. Obsérvese que tanto en las respuestas procedentes de los esquemas A como de los B se establece la relación entre las variables $n$ y $f(n)$, pero mientras en las de la clase A se aproxima al objeto matemático $f(n)=a n+b$, en las de la clase $\mathrm{B}$, la respuesta se aleja de tal objeto.

Recordemos que el número de alumnos que utilizaron la estrategia visual en las cuatro cuestiones de las dos tareas quedó reducido a 40 de un total de 373 (11\%). La tabla VIII muestra el número de alumnos que realizan una transición particular entre ambas tareas, según los esquemas de acción utilizados.
Tabla VIII

Transición entre las dos tareas.

\begin{tabular}{cc} 
Tipo & Alumnos \\
\hline De A a A & 24 \\
\hline De B a B & 1 \\
\hline De A a B & 1 \\
\hline De B a A & 14 \\
\hline Total & 40 \\
\hline
\end{tabular}

En primer lugar, observamos que 25 alumnos $(62,5 \%)$ usan el mismo tipo de respuesta al pasar de la tarea de la escalera a la del árbol de Navidad. Por otro lado, los restantes 15 alumnos $(37,5 \%)$ modifican el tipo de respuesta al abordar el árbol de Navidad. De la tabla VIII se infiere que 38 alumnos (95\%) mantienen o cambian a una respuesta de tipo $\mathrm{A}$, mientras que 2 alumnos $(5 \%)$ persisten o cambian a una respuesta de la clase B. Luego, es clara una tendencia hacia las respuestas de clase A, que están más próximas al objeto matemático.

Un estudio más detallado del comportamiento de los alumnos que utilizan el dibujo y su comparación con otros que no lo utilizan sería de gran ayuda y entra dentro de nuestro proyecto de investigación. Algunos de tales resultados han sido publicados en García Cruz y Martinón (1997a) y en esencia corresponden a determinadas acciones sobre la sucesión numérica y al doble papel que juega el dibujo, siendo en algunos casos, el ámbito donde se desarrolla el proceso de generalización y en otros un medio para comprobar la validez de los invariantes establecidos que actúan únicamente sobre la sucesión numérica.

\section{Consideraciones didácticas}

Creemos que algunas consideraciones de tipo didáctico se pueden derivar de este estudio. En el proceso de enseñanza y aprendizaje, especialmente para el profesor, es importante el medio para conducir a los alumnos hacia un objetivo o meta curricular. En situaciones como las aquí planteadas subyace una entidad conceptual y estas situaciones pueden ser punto de partida para alcanzar tal concepto.

Hemos destacado la importancia de las acciones realizadas por el alumno sobre el dibujo, como punto de partida y génesis de la generalización. Desde un punto de vista didáctico resulta también importante establecer condiciones para la acción, entendidas como requerimientos, exigencias o limitaciones a las que se someten esas acciones. No se trata de que el profesor imponga esas condiciones, sino que deben surgir de forma negociada en el aula, tras la exposición y discusión de las respuestas libres que hayan dado los alumnos. La importancia de estas condiciones es obvia, pues hay acciones que no conducen a un determinado objetivo, mientras que otras sí lo hacen.

Hemos visto que los alumnos que usan la estrategia visual, en situación libre, sin instrucciones concretas, 
actúan sobre el dibujo de dos maneras bien diferentes, las señaladas en los esquemas A (con diferencia) y B (sin diferencia). Pues bien, creemos que las respuestas de tipo A establecen claramente las condiciones para la acción a desarrollar. Si el profesor quiere llevar a los alumnos hacia el concepto de función lineal y parte de situaciones como las utilizadas en este trabajo u otros similares, deberá reconducir las acciones de los alumnos, si fuera necesario, al esquema $\mathrm{A}$.

En el esquema A, el elemento variable de la acción está directamente relacionado con la diferencia $a$. El elemento invariable puede estar directamente relacionado con $b$, como así ocurre en las modalidades L11 para la escalera y L12 para el árbol de Navidad o, de forma indirecta, como ocurre en las otras modalidades que tienen en común las respuestas de tipo $\mathrm{A}$. Si el alumno opta por una modalidad encuadrada bajo el esquema B, se pueden introducir símbolos literales y mediante las correspondientes simplificaciones obtener expresiones propias del esquema A. Este proceso debería realizarse en presencia del dibujo y relacionando las operaciones y simbolismos con el dibujo, con el fin de darles significado. Por ejemplo, la conversión de la expresión $n+n+$ $(n-1)+(n-1)+1$ (que se corresponde con la modalidad L33 del árbol) en la expresión $4 n-1$ (propia de la modalidad L12 del árbol) nos lleva a contemplar el dibujo de forma distinta, dentro del esquema A, que está más próximo al objeto $f(n)=a n+b$.

Para ampliar detalles sobre la intervención en el aula remitimos, al lector interesado, a los trabajos de García Cruz y Martinón (1997b y 1998).

\section{CONCLUSIÓN}

La investigación expuesta en este trabajo aporta una descripción y concreción de la estrategia visual, sus esquemas generales y modalidades particulares, elaborada por los alumnos, de forma espontánea, al enfrentarse con tareas de generalización lineal.

\section{REFERENCIAS BIBLIOGRÁFICAS}

BIGGS, J.B. y COLLIS, K.F. (1982). Evaluating the quality of Learnig. Nueva York: Academic Press.

CASTRO,E.(1995). Exploración de patrones numéricos mediante configuraciones puntuales. Estudio con escolares del primer ciclo de secundaria (12-14 años). Granada: Comares.

DCB (1989). Diseño curricular base. Educación secundaria obligatoria II. Madrid: Ministerio de Educación y Ciencia.

DÖRFLER, W. (1991). Forms and Means of Generalization in Mathematics, en Bishop, A. et al. (eds.), Mathematical Knowledge: Its Growth Through Teaching. Kluwer Academic Publishers.
Hemos puesto de manifiesto el papel importante que juega el dibujo que acompaña a las tareas en el desarrollo de tal estrategia. El modelo desarrollado por Dörfler (1991), aplicado al análisis de tal estrategia, se ha mostrado muy útil en la explicación de cómo realiza el alumno la construcción de la generalización. Las acciones mentales introducidas por los alumnos en los objetos, consistentes en la separación de tales objetos en elementos variables e invariables, llevan al establecimiento de ciertas relaciones invariantes, que conducen a los alumnos a dar respuesta a lo que se les pide. En relación con la práctica pedagógica, concluimos que los esquemas A parecen más adecuados, por su proximidad estructural al concepto matemático subyacente, para fijar las condiciones de la acción. Respondemos así a la cuestión 1 que nos planteamos al inicio.

La cuestión 2 tiene la siguiente respuesta: en líneas generales, existe una tendencia mayor a usar la estrategia visual al aumentar el nivel académico y la edad, aunque los datos no son concluyentes. Lo que sí parece claro es que, con el tiempo, los alumnos olvidan la instrucción recibida sobre sucesiones aritméticas, debido quizás a que la expresión aprendida y la metodología empleada no fue la adecuada. Así, la expresión $f(n)=$ $f(1)+d(n-1)$ se percibe más como una fórmula para un cálculo específico que como la relación existente entre el par de parámetros $a$ y $b$ y las variables $n$ y $f(n)$, y es aquella percepción la que se fomenta en la metodología aplicada por el profesor y los libros de texto más usados en España.

Las más modernas orientaciones curriculares que contemplan nuevos enfoques y contenidos permiten establecer conexiones entre elementos del currículo que hasta ahora quedaban aislados. Tal es el caso de las pautas numéricas, las sucesiones y las funciones. Los esquemas cognitivos que desarrollan los alumnos en situación de exploración libre pueden llevar, utilizados adecuadamente por el profesor, a establecer esas conexiones y desarrollar una comprensión más profunda entre conceptos que hasta ahora se presentaban aislados.

DUBINSKY, E. y HAREL, G. (1992). The nature of the process conception of function, en Dubinsky y Harel (eds.), The Concept of Function, Aspects of Epistemology and Pedagogy. MAA.

GARCÍA CRUZ, J.A., y MARTINÓN, A. (1996a). Modelos y estrategias en problemas de generalización lineal, en Dorta, J.A. et al. (eds.), 25 años de matemáticas en la Universidad de La Laguna, pp. 297-307. Universidad de La Laguna.

GARCÍA CRUZ, J.A., y MARTINÓN, A. (1996b). Personal Strategies of Generalization in Linear Generalizing Problems, en Puig, L. y Gutiérrez, A. (eds.), Proceedings of 
the 20th International Conference for the Psychology of Mathematics Education, 1, p. 174. Universitat de València.

GARCÍA CRUZ, J.A. y MARTINÓN, A. (1997a). Actions and Invariant Schemata in Linear Generalizing Problems, en Pehkonen, E. (ed.). Proceedings of the 21 st International Conference for the Psychology of Mathematics Education, 2, pp. 289-296. Univerity of Helsinki.

GARCÍA CRUZ, J.A., y MARTINÓN, A. (1997b). A Teaching experience with linear pattern and sociomathematical norms, en Abrantes, P. y Porfirio, J. (eds.), Proceedings of the 49 CIEAEM. Universidad de Setúbal. (Pendiente de publicar.)

GARCÍA CRUZ, J.A., y MARTINÓN, A. (1998). Interacción y construcción significativa del conocimiento: notas teóricas y una práctica educativa. UNO Revista de Didáctica de las Matemáticas. (Pendiente de publicar.)

MASON, J.L. y STACEY, K. (1982). Thinking Mathematically. Addison-Wesley Publishing Company. Trad. cast., 1988. Pensar matemáticamente. Madrid: Editorial Labor y Ministerio de Educación y Ciencia.

NCTM. (1989). Curriculum and Evaluation Standards for School Mathematics. National Council of Teachers of Mathematics, Reston.

ORTON, A. y ORTON, J. (1994). «Students» Perception and use of pattern and generalization, en da Ponte, J.P. y Matos, J.F. (eds.), Proceedings of the Eighteenth International Conference for the Psychology of Mathematics Education, 3, pp. 407-414. Universidad de Lisboa.

ORTON, A. y ORTON, J. (1996). Making sense of Children Patterning, en Puig, L. y Gutiérrez, A. (eds.), Proceedings of the Twentieth International Conference for the Psychology of Mathematics Education, 4, pp. 83-90. Universidad de Valencia.

PHILLIPS, E. (ed.) (1991). Patterns and Functions. Addenda Series, grades 5-8. National Council of Teachers of Mathematics, Reston.

POLYA, G. (1966). Matemáticas y razonamiento plausible. Madrid: Tecnos.

REAL DECRETO 1345/1991. Curriculum de la Enseñanza Secundaria Obligatoria. BOE, 220. Madrid.

REDDEN, E. (1994). Alternative Pathways in the Transition form Arithmetic Thinking to Algebraic Thinking, en da Ponte, J.P. y Matos, J.F. (eds.), Proceedings of the Eighteenth International Conference for the Psychology of Mathematics Education, 4, pp. 89-96. Universidad de Lisboa.

SWAN, M. (ed.) (1984). Problems with Patterns and Numbers. Join Matriculation Board. Shell Centre for Mathematical Education. Birmingham. Trad. cast. Problemas con pautas y números. Bilbao: Servicio Editorial del País Vasco.

STACEY, K. (1989). Finding and Using Patterns in Linear Generalising Problems. Educational Studies in Mathematics 20, pp. 147-164.

TAPLIN, M. (1995). Spatial Patterning: A Pilot Study of Pattern Formation and Generalization, en Meira, L. y Carraher, D. (eds.), Proceedings of the Nineteenth International Conference for the Psychology of Mathematics Education, 3, pp. 42-49. Recife: Universidade Federal de Pernambuco.

[Artículo recibido en septiembre de 1997 y aceptado en julio de 1998.] 